\title{
Propuesta de una red de cooperación intersectorial para gestionar prácticas profesionales universitarias en el estado de Falcón, Venezuela
}

\author{
Freddy Marín-González¹, Judith Pérez-González ${ }^{2}$, Alexa Senior-Naveda', Ana J. Paredes-Chacín ${ }^{3}$ \\ (1) Departamento de Humanidades, Universidad de la Costa, Atlántico - Colombia (correo-e: fmarin1@cuc.edu.co; \\ asenior@cuc.edu.co) \\ (2) Complejo Académico "El Sabino". Dpto. de Gerencia, Universidad Nacional Experimental "Francisco de Miranda" \\ UNEFM, Falcón - Venezuela (correo-e: judithmpg@correo.unefm.edu.ve) \\ (3) Universidad Autónoma de Occidente, Cali - Colombia (correo-e: ajparedes3@uao.edu.co)
}

Recibido Mar. 5, 2020; Aceptado May. 6, 2020; Versión final Jun. 22, 2020; Publicado Oct. 2020

\begin{abstract}
Resumen
Este artículo propone la conformación de una red de cooperación para la gestión de prácticas profesionales universitarias en el contexto relacional universidad-gobierno-empresa. Se fundamenta en un enfoque racionalista y la aplicación de métodos de razonamiento lógico inferencial a través de las técnicas de análisis de fuentes documentales, deducción y abducción, desde los aportes de las teorías de la cooperación, redes y currículo. La población la conforman actores clave de los sectores involucrados ubicados en la Península de Paraguaná, estado Falcón, Venezuela. Se les aplicó un cuestionario cuyos resultados evidencian disposición a formar parte de la red y a cumplir acuerdos y objetivos propuestos. Hay existencia de actores con tipologías destacables los cuales representan una fuente importante de información. Como conclusión se valida que los flujos relacionales entre sectores propician la creación de redes formales de cooperación fortalecidas desde los aportes de cada nodo de la red.
\end{abstract}

Palabras clave: redes de cooperación; prácticas profesionales; intersectorialidad; gestión; relación universidad empresa

\section{Proposal for an inter-sectorial cooperation network to manage university professional practices in the Falcón region, Venezuela}

\begin{abstract}
This article proposes the formation of a cooperation network for the management of university professional practices in the context of the relationships between university, government, and private sectors. It is based on a rationalistic approach and on the application of inferential logical reasoning methods. This is performed through the analysis of documentary sources (deduction and abduction techniques) on cooperation, networks, and curriculum theories. The population is composed of key actors in the university, government, and private sectors from the Paraguaná Peninsula (Falcón state) in Venezuela. A questionnaire was applied and the results show willingness to be part of the network and to comply with agreements and proposed objectives. There are actors with outstanding typologies that represent an important source of information. In conclusion, the present study validates that the relationships between sectors favor the creation of formal cooperation networks that are strengthened by the contributions from each node in the network.
\end{abstract}

Keywords: cooperation networks; professional practices; inter-sectoriality; management; business university relationship 


\section{INTRODUCCIÓN}

La globalización implica una serie de desafíos y oportunidades para las organizaciones, ya que para que estas puedan operar de forma eficaz en un mundo globalizado es necesario buscar, conformar y fortalecer alianzas, asociaciones y redes con otras entidades como una vía de complementar capacidades, experiencias y conocimientos. Larner (2015), define la red como una estructura formal o informal conformada por personas que, utilizando diferentes canales de comunicación, comparten responsabilidades, conocimientos, trabajo, proyectos, recursos, documentos, productos y servicios, con el fin de crecer como equipo, como colectivo y lograr objetivos predeterminados. Las redes implican desarrollos complejos de acción recíproca que se retroalimentan y, a su vez, representan un medio para obtener recursos sustantivos para las organizaciones. Por su parte, Sánchez et al., (2014), señalan que estas representan las relaciones de actores sociales que participan en el proceso de creación e intercambio de conocimiento; concebidas como un grupo de personas y sus vínculos de conocimiento que interactúan e intercambian información sobresaliente como parte de sus relaciones formales o informales.

Emergen redes de cooperación como asociaciones de interesados que tienen como objetivo la consecución de resultados acordados conjuntamente a través de la colaboración mutua, la vinculación se sustenta en una estructura horizontal de coparticipación, colaboración y corresponsabilidad de cada uno de sus asociados con relación a un plan de acción Sebastián (2000). Para Hernández y Chumaceiro (2018), la participación resulta clave para la integración de actores como vía de fortalecimiento mutuo. Al respecto Gutiérrez y Narváez (2017), definen las redes de cooperación como sistemas complejos y dinámicos, de relaciones e interacciones, establecidos entre actores sociales de carácter público y privado, para alcanzar objetivos comunes, a través de la cooperación y el intercambio de información, recursos, bienes y servicios. MarínGonzález et al., (2019), señalan como condicionante para el establecimiento de redes de cooperación a la propia realidad y sus problemas; en contextos complejos se requieren abordajes interdisciplinarios para fortalecer la creación de equipos; ello supone, la participación activa de los actores en la búsqueda de la integración intersectorial en el ámbito local, regional, nacional e internacional.

Ante esta realidad, organizaciones como la universidad debe reivindicar su postura ética ante la ciencia, tecnología e innovación, estimulando nuevas modalidades de vinculación con sectores como gobierno, empresas y comunidades para formar profesionales idóneos a las nuevas exigencias sociales, tal como lo destacan Bornal (2009) y Marín-González et al., (2018), en el marco de la sociedad globalizada las instituciones universitarias como generadoras de conocimiento, para enfrentarse a escenarios de cambios, necesitan revalorizarse desde su quehacer ético, académico, investigativo y extensionista para elevar la calidad de los egresados; se requiere una formación integral centrada en un perfil por competencias científico y tecnológicas; habilidades para desempeñarse proactivamente en un mercado laboral más especializado; al respecto Gardner y Tyson (1994), refieren que los estudiantes necesitan oportunidades para ocuparse en experiencias laborales reales que les demanden aplicar sus aprendizajes en sitios de ejercicio profesional.

Por consiguiente, el objetivo de este artículo es la propuesta de un diseño de red de cooperación para la gestión efectiva de las prácticas profesionales desde los actores sociales universidad - gobierno -empresa (Red CUGE), con la intención de contribuir con el crecimiento y desarrollo interorganizacional. La red, tendrá un radio de acción local en la Península de Paraguaná, la cual está ubicada al norte del estado Falcón, en Venezuela y la cual está conformada por tres Municipios: Carirubana, Los Taques y Falcón. Los actores identificados lo conforman las universidades públicas, las entidades de gobierno y las empresas de producción de bienes y servicios que hacen vida en la Península de Paraguaná.

En correspondencia a lo expuesto es necesario explicar las redes de cooperación entre el sector universitario, sector gobierno y sector empresarial en relación a la gestión de las prácticas profesionales, al igual que caracterizar la gestión de las prácticas profesionales en el marco de la intersectorialidad; por lo cual se analizan constructos teóricos referidos al análisis de redes sociales, redes de cooperación y prácticas profesionales. Se aborda la cooperación desde la dimensión de las prácticas profesionales con la aplicación de un instrumento, prestando atención a la dinámica funcional de los sectores: gobierno - universidad empresa, involucrados en la propuesta.

Todo ello permitió la construcción de un diseño de red de cooperación donde se logra identificar su planificación estratégica (misión, visión, valores, objetivos, estructura organizativa, sistema de gobernanza), actores principales, productos a ofrecer, procesos involucrados, indicadores de funcionamiento los cuales permiten en un momento dado hacer los ajustes necesarios para que la red tenga un mejor desempeño. Con el diseño propuesto se espera contribuir al fortalecimiento de los perfiles profesionales y al incremento de la competitividad intersectorial. La propuesta de la red de cooperación se fundamenta en un enfoque racionalista con aplicación de métodos de razonamiento lógico inferencial a través de las técnicas de análisis de fuentes documentales. 


\section{OTROS ANTECEDENTES}

Las prácticas profesionales son un eje fundamental en la formación de profesionales universitarios; representan un medio articulador para la cooperación entre actores sociales que inciden en sus procesos. En opinión de Peña et al., (2016), es un componente curricular orientado, en principio, a forjar el perfil de egreso desde el hacer, es decir, a partir de la ejecución de labores profesionales bajo la orientación académica y experta de tutores; que además tiene el efecto de validar en un doble sentido la formación recibida; primero, para el propio sujeto (pasante) quien se hace consciente e internaliza el manejo, implementación y alcance de sus propios conocimientos; y segundo, para la propia institución educativa que lo envía, quien puede verificar la pertinencia social de los contenidos académicos que imparte en la solución de los problemas del entorno en el cual opera. En este sentido la UNESCO (1998), ratifica la necesidad que las universidades e institutos y colegios universitarios trabajen de cara al contexto y al momento histórico con sentido de pertinencia, de apertura a nuevas formas de aprendizaje que se correspondan con las características de vida del trabajador.

En el ámbito de las relaciones de cooperación, Gulati (1998) refiere que las organizaciones constituyen vínculos cooperativos para acceder a recursos, capacidades, aprendizajes que son esenciales para lograr sus objetivos, pero que están, al menos en parte, bajo el control de otras organizaciones en su medio ambiente. Estos vínculos propician la creación de redes interorganizacionales; para Espinoza y MarínGonzález (2019) constituyen una cantidad de organizaciones que, en forma agrupada o individual, se interrelacionan por objetivos específicos, conducida por un flujo de información y el cumplimiento de procesos y actividades integradas.

Se toma en consideración lo expresado por Aguirre (2014) cuando establece que para definir a una red, primero se debe determinar el/las relación/es contextualizada que interesa estudiar, esto habilita para definir el límite de la red y determinar el conjunto finito de actores involucrados (individuales, grupales 0 institucionales), así limitar empíricamente la red y seleccionar la información necesaria para su conformación; se concentra en la red de relaciones, las posiciones funcionalmente diferenciables dentro de éstas, sus mecanismos dinámicos de adaptación, sus flujos y transacciones, entre otras; implica, tal como señalan Romero y Zabala (2018) procesos de planeación de carácter estratégico y contextual. En cuanto al análisis de redes Bravo et al., (2013) y Madeo y Mocceni (2020), destacan la importancia de describir y estudiar las estructuras relacionales que surgen cuando diferentes organizaciones o individuos interaccionan, se comunican, coinciden, colaboran entre otras; a través de diversos procesos o acuerdos, que pueden ser bilaterales o multilaterales; de este modo la estructura que emerge de la interrelación se traduce en la existencia de una red social.

Los principales aportes devienen de teóricos como Wasserman y Faust (1994) quienes teorizan acerca del diseño y evaluación de redes; sus postulados son retomados por Sebastián (2000), Aguilar-Gallegos et al., (2016), Espinoza y Marín-González (2019), quienes sugieren para el diseño o evaluación de redes propiedades, como la densidad o relación entre el número de lazos presentes y de lazos posibles; la existencia de nodos dominantes en la red; al igual que la transitividad o capacidad de un actor para llegar a otros. Ahora bien, todos estos elementos constituyen indicadores de su funcionamiento, lo que permitiría en un momento dado hacer los ajustes necesarios para que la red tenga un mejor desempeño. La representación que se tenga de una red por tanto sirve de guía para definir procesos, identificar recursos, declaración de normas, entre otros aspectos, por lo que el tener claro en qué consiste cada uno de ellos es vital para su gestión y reorganización.

Para Freeman (2004), es importante destacar no solo el componente estructural en el diseño de redes, sino las características relacionales y funcionales de los actores participantes, donde la reciprocidad, homogeneidad y heterogeneidad, compromiso, cadena de comunicación, confianza, vínculos precedentes y actuales, entre otros condicionan el comportamiento y los mecanismos a través de los cuales las organizaciones acceden y explotan las fuentes de conocimiento. Marín-González et al., (2019) refieren que el perfil operativo de una red expresa flujos de comunicación, intercambio y transacción entre actores; normas y valores que condicionan conductas y formas de comportamiento. Donde se evidencian posiciones funcionalmente diferenciables trabajando en temas específicos, mediante la compartición de información, conocimiento, infraestructura, costos, recursos.

En relación a la trama transaccional y tomando en consideración sus atributos se busca apuntalar estrategias que tributen a: a) búsqueda de mecanismos que permitan flexibilizar las normas administrativas que puedan inhibir la vinculación universidad - gobierno - empresa; b) Asegurar al pasante espacios de ejercicio profesional que le permitan integrar y aplicar las competencias adquiridas, de acuerdo al perfil de egreso y nivel de formación; c) Propiciar mecanismos de vinculación y cooperación entre los actores de la red, 
impulsando la participación activa entre grupos de interés en relación a la necesidad de pasantes333 universitarios para la solución de problemas específicos; d) Establecer mecanismos que permitan el fortalecimiento de los estudiantes en capacidades, para responder a los requerimientos del desarrollo local; e) Contribuir al diseño de programas universitarios, específicamente en relación al perfil de egreso de acuerdo a las exigencias del mundo actual; f) Instaurar una plataforma de telecomunicaciones que permita una fluidez de comunicaciones entre los actores involucrados de manera que puedan intercambiar conocimiento en relación a las mejores prácticas del proceso de gestión; g) fortalecer mecanismos de vinculación y cooperación entre los actores de la red, impulsando la participación activa entre grupos de interés en relación a la necesidad de pasantes universitarios para la solución de problemas específicos.

\section{METODOLOGÍA}

El componente metodológico, parte de un enfoque epistemológico racional crítico, donde se busca dar respuestas valiéndose de razonamientos encadenados cuyas conclusiones son comparadas con la realidad. Al respecto, Padrón (1992), establece una manera de generar conocimiento por medio del plano de las ideas, a través de la teoría, donde prevalece un estilo racional. Dada la finalidad de la investigación es necesario comprender los principios, fundamentos, elementos constitutivos de una red de cooperación, así, como también interpretar los procesos, interrelaciones, percepciones y acciones relacionados con la gestión de las prácticas profesionales, para lo cual se consideran los principales postulados teóricos que caracterizan la cooperación, el diseño de redes, redes interorganizacionales, currículo, prácticas profesionales; lo que permite establecer conexiones entre las distintas categorías y en correspondencia con el contexto del cual forman parte. A partir de lo expuesto se genera un sistema de relaciones teóricas que constituye junto al tratamiento directo de las unidades de análisis, fundamentos de aproximación a la conformación de la red de cooperación universidad - gobierno - empresa (Red CUGE) que contribuya a una mejor gestión de las prácticas profesionales en las universidades.

En relación al diseño se enmarca en un componente propositivo de naturaleza heurística, tomando como base un abordaje de la realidad, donde a través de la construcción de un cuestionario de cincuenta (50) preguntas cerradas a escala múltiple, estructurado en nueve (09) secciones a considerar: Aspectos Legales, Administrativa, Relaciones Laborales, Profesionales, Didácticos, Personales, Actores que integran la red, Capacidad y Vínculos de Cooperación; permitió determinar los sectores intervinientes, al igual que la evidencia necesaria para la conformación de la red propuesta.

En tal sentido la población o universo de estudio quedó integrada por catorce (14) personas, representantes de los diferentes sectores involucrados en la investigación, todos ellos seleccionados a través de un muestreo no probabilístico intencional (con conocimiento en funciones gerenciales y procesos de prácticas profesionales) quedando conformada así: a) Sector Universitario: Por un coordinador del proceso de prácticas profesionales en las universidades: Universidad del Zulia (LUZ), Universidad Nacional Experimental Francisco de Miranda (UNEFM), Universidad Nacional Experimental de la Fuerza Armada Nacional Bolivariana (UNEFA), Universidad Bolivariana de Venezuela (UBV), Universidad Politécnica Experimental Libertador (UPEL), Universidad Nacional Abierto (UNA). b) Sector Empresarial: Un representante de las diferentes cámaras que hacen vida en la Península de Paraguaná, Cámara Petrolera, Cámara de la Industria, Cámara de Comercio, Cámara de la Construcción. c) Sector Gobierno: un representante de la Gobernación del Estado Falcón y un representante de las alcaldías del municipio Carirubana, Los Taques y Falcón que conforman la Península de Paraguaná

Para la validez se utilizó el juicio de expertos, por lo cual se suministró el cuestionario a cinco (05) docentes de diferentes instituciones universitarias tanto nacionales como internacionales, del área específica de la investigación, donde dictaminaron acerca la pertinencia, coherencia, relevancia y exactitud de cada uno de los indicadores propuestos con una valoración de Excelente, Bueno, Regular, y Deficiente. La confiabilidad del instrumento se determinó por el Coeficiente del Alpha de Cronbach, con base a una muestra representativa de cinco individuos que trabajan en los sectores objetos de estudio, los resultados arrojados se tabularon utilizando el software Microsoft Excel y demostraron que el instrumento es altamente confiable al obtener como coeficiente 0,867 . Para el procesamiento de los datos se utilizó la estadística descriptiva e inferencial para generalizar la tendencia e inferir conclusiones de la población en el estudio, este proceso se realizó a través del software estadístico Statistical Package for the Social Sciences (SPSS).

La conformación de la red, se fundamenta en una acción conjunta y coordinada de los actores involucrados localmente; se busca a través de la complementariedad optimizar sus recursos para la obtención de las mejores prácticas en el logro de los objetivos planteados; sus planes de acción no son cortoplacistas, por lo que se requiere para su consolidación horizontes de tiempo de mediano y largo plazo, y una estructura sólida de comunicación e información. En función a lo anterior para su diseño se toman en cuenta las etapas identificadas en la Tabla 1. 
Tabla 1: Etapas del Diseño.

\begin{tabular}{|c|c|c|c|}
\hline $\begin{array}{l}\text { Categorías de la } \\
\text { Investigación }\end{array}$ & $\begin{array}{l}\text { Constructos } \\
\text { Esenciales }\end{array}$ & $\begin{array}{l}\text { Etapas del } \\
\text { Diseño }\end{array}$ & Principales Actividades \\
\hline $\begin{array}{l}\text { Gestión de Prácticas } \\
\text { Profesionales }\end{array}$ & $\begin{array}{l}\text { Estructura de } \\
\text { funcionamiento. } \\
\text { Condiciones de } \\
\text { relación. } \\
\text { Lineamientos. }\end{array}$ & Negociación & $\begin{array}{l}\text { Diagnóstico de la relaciones de cooperación } \\
\text { Universidad - Gobierno - Empresa en la gestión de } \\
\text { prácticas profesionales - Análisis de contenido } \\
\text { Determinación los actores que participan en la red }\end{array}$ \\
\hline $\begin{array}{l}\text { Redes de } \\
\text { Cooperación }\end{array}$ & $\begin{array}{l}\text { Actores sociales } \\
\text { Actores claves } \\
\text { Propósito de la red } \\
\text { Vínculos } \\
\text { Atributos de los } \\
\text { vínculos } \\
\text { Estructura } \\
\text { Dinámica funcional }\end{array}$ & Compromiso & $\begin{array}{l}\text { Definición del Marco Filosófico de la Red CUGE: } \\
\text { Misión- Visión -Valores - Objetivos-Gobernanza- } \\
\text { Construcción de la estructura Morfológica de la Red. } \\
\text { Interpretación y tratamiento de datos - Información } \\
\text { sistematizada - Software UCINET. Versión Software } \\
\text { UCINET Versión } 6.667 \text { (Borgatti, Everett y Freeman, } \\
\text { 2002) }\end{array}$ \\
\hline
\end{tabular}

\section{RESULTADOS Y DISCUSION}

Para la configuración y estructura del diseño, se plantean las siguientes etapas organizadas secuencialmente: Diagnóstico de las relaciones de cooperación Universidad - Gobierno - Empresa, Determinación de los actores participantes de acuerdo a su perfil de competencias, Definición del marco filosófico de la red CUGE, Construcción de la estructura Morfológica de la Red. Interpretación y tratamiento de datos - Información sistematizada - Software UCINET Versión Software UCINET Versión 6.667.

\section{Diagnóstico de la relaciones de cooperación}

El análisis obtenido lleva a considerar a las prácticas profesionales como actividad tradicional de vinculación de las universidades con otras organizaciones; representan un mecanismo facilitador de las relaciones de cooperación con el sector gobierno y empresarial de forma equilibrada; en lo referido al momento, duración, objetivos, contenidos formativos, sistema de evaluación, supervisión entre otros. Pérez González et al., (2019), destacan la necesidad de establecer vínculos entre la universidad y el sector empresarial, conformando redes de cooperación interorganizacionales que permitan la gestión de las pasantías industriales, por lo que se deben establecer nuevas estrategias que ayuden a fortalecer la intensidad relacional.

Se desprende que la colaboración universidad empresa puede surgir a partir de una actividad básica de las universidades (la capacitación), existiendo una percepción entre los empresarios de que los recursos humanos producidos por las instituciones de educación superior están por debajo de los requerimientos de la industria. Se plantea la necesidad de fortalecer la producción científica en las universidades, como mecanismo facilitador en la relación universidad entorno, al igual que una cultura organizacional con base al trabajo grupal cooperativo para el desarrollo de redes de cooperación. Al respecto Barrios-Hernández y Olivero-Vega (2020), refieren que al trasladar el conocimiento fuera de las universidades, mediante estrategias que le permiten la interacción entre sus actividades sustantivas con instituciones del medio externo se puede generar beneficios mutuos.

El análisis devela una clara disposición de los actores involucrados a: formar parte de la red, cumplir acuerdos alcanzados a través del consenso, respetar normas y conseguir el aprovechamiento de los recursos técnicos, tecnológicos o académicos, igualmente se identifican rasgos de un perfil de desempeño centrado en habilidades, destrezas, conocimiento en campos particulares para los actores involucrados. Se fundamenta la actuación en una estructura matricial, donde se evidencian los procesos básicos que se deben llevar en la red, la cual permite a los actores trabajar de manera flexible, un flujo de comunicación de manera constante, al igual que adaptarse a los999 cambios y demandas de sus clientes para satisfacerlos de una manera más oportuna. Igualmente se evidencia la necesidad de las organizaciones de reevaluar su cultura y objetivos que le permitan potenciar y dinamizar las relaciones con su entorno, para estar a la par de los cambios exigidos por la sociedad. Desde la perspectiva de Tubin y Levin-Rozalis, (2008) es difícil pensar en la actualidad de una organización, en especial que provee servicios, que no opere en cooperación o en red con otras organizaciones. 


\section{Determinación de los actores participantes}

En la propuesta de red CUGE, se identifican actores de ámbito local, para lo cual se realizó un análisis de los atributos de las organizaciones participantes en lo referente a: actividad, capacidad, trayectoria y posicionamiento lo que permite realizar perfiles de competencia específico que coadyuva positivamente a su funcionamiento. A continuación en la Tabla 2, se muestran los atributos más resaltantes.

Tabla 2: Perfil de los actores participantes de la red CUGE

\begin{tabular}{|c|c|c|c|}
\hline & Actores & Rol del Actor & Participación en la red \\
\hline 1 & $\begin{array}{l}\text { Coordinación de Pasantías de la } \\
\text { UNEFM. }\end{array}$ & \multirow[t]{6}{*}{ Conocimiento } & \multirow{6}{*}{$\begin{array}{l}\text { Constituyen el eje central de la red sobre el cual giran las } \\
\text { actividades de prácticas profesionales. Teniendo a cargo } \\
\text { coordinar todas las actividades del proceso, por medio } \\
\text { de unidades, procesos y recursos, para garantizar que las } \\
\text { prácticas se lleven de acuerdo al perfil de egreso y los } \\
\text { objetivos de productividad planteados sean alcanzados. }\end{array}$} \\
\hline 2 & $\begin{array}{l}\text { Sección Departamental de } \\
\text { Prácticas Profesionales de LUZ }\end{array}$ & & \\
\hline 3 & $\begin{array}{l}\text { Coordinación de Prácticas } \\
\text { UNEFA }\end{array}$ & & \\
\hline 4 & $\begin{array}{l}\text { Coordinación de Pasantías de la } \\
\text { UBV }\end{array}$ & & \\
\hline 5 & Coordinación de Prácticas UNA & & \\
\hline 6 & Coordinación de Prácticas UPEL & & \\
\hline 7 & $\begin{array}{l}\text { Cámara Petrolera. Capitulo } \\
\text { Falcón }\end{array}$ & Receptor & $\begin{array}{l}\text { Principal recaudador de pasantes dentro de la red, } \\
\text { proporcionando capacitación técnica y asesoramiento } \\
\text { tecnológico con posibilidades de favorecer las soluciones } \\
\text { de problemas de interés local. }\end{array}$ \\
\hline 8 & Cámara de la Construcción & \multirow[t]{3}{*}{ Receptor } & \multirow{3}{*}{$\begin{array}{l}\text { Agrupa a todos los comerciantes de la zona, dentro de la } \\
\text { red son fuentes de contacto primario que permiten la } \\
\text { colocación de pasantes universitarios en los centros de } \\
\text { práctica. Adicionalmente establece políticas sectoriales de } \\
\text { intercomunicación continua para solicitar, acceder y } \\
\text { recibir los servicios que en materia de pasantes requiere } \\
\text { la Península de Paraguaná, además de ser fuentes de } \\
\text { asesoramiento tecnológico para la solución de problemas } \\
\text { locales. }\end{array}$} \\
\hline 9 & Cámara de industriales & & \\
\hline 10 & Cámara de Comercio & & \\
\hline 11 & Gobernación del Estado Falcón & $\begin{array}{l}\text { Mediador - } \\
\text { Facilitador }\end{array}$ & $\begin{array}{l}\text { Constituido por el representante del gobierno estadal, su } \\
\text { finalidad dentro de la red es de apoyo, y coordinación de } \\
\text { los representantes del gobierno local en la red, así como } \\
\text { generar políticas y estrategias de vinculación entre los } \\
\text { actores involucrados, al igual que la promoción de la red } \\
\text { CUGE, como mecanismo de fortalecimiento de formación } \\
\text { de estudiantes universitarios. }\end{array}$ \\
\hline 12 & Alcaldía del Municipio Carirubana & \multirow[t]{3}{*}{$\begin{array}{l}\text { Mediador - } \\
\text { Facilitador }\end{array}$} & \multirow{3}{*}{$\begin{array}{l}\text { Conformado por los representantes de organismos del } \\
\text { gobierno municipal, su finalidad en la red es detección las } \\
\text { necesidades prioritarias de la península de Paraguaná, } \\
\text { dentro de las cuales los pasantes universitarios se puedan } \\
\text { incorporar, igualmente poseer capacidad para obtener } \\
\text { recursos potenciales y fuentes de financiamientos para el } \\
\text { mejor funcionamiento de la red. }\end{array}$} \\
\hline 13 & Alcaldía del Municipio los Taques & & \\
\hline 14 & Alcaldía del Municipio Falcón & & \\
\hline
\end{tabular}

Aunado a lo anterior, se identifican aquellas partes interesadas (stakeholders o grupos de interés) relacionadas con la gestión de la red CUGE, con quienes ésta interactúa. Destacan entre los stakeholders estudiantes, profesores, personal administrativos, autoridades universitarias, entes gubernamentales, empresas, empleadores, entre otros grupos de interés que permiten la conformación de los nodos de la red y describen la dinámica de flujos relacionales entre actores.

\section{Definición del Marco Filosófico de la Red CUGE}

Tiene como misión promover en el ámbito interorganizacional e intersectorial la gestión de relaciones de cooperación, con base en el desarrollo de prácticas profesionales donde los estudiantes universitarios desplieguen competencias acordes a un alto nivel de desempeño. Su Visión es constituir una red de cooperación intersectorial e interorganizacional, donde la gestión de las prácticas profesionales de los estudiantes universitarios, sea el proceso medular que garantice la interconectividad y flujos relacionales entre sus componentes estructurales y funcionales. 


\section{Construcción de la estructura Morfológica de la Red}

En relación a la estructura de la red, es importante considerar la representación abstracta de la red, al respecto se requiere un conjunto de métodos y conceptos analíticos distintos de los métodos estadísticos y análisis de datos tradicionales Wasserman y Faust (1994). Desde la perspectiva de Maya-Jariego y Holgado (2015) y Herrera-Núñez y González-Campos (2019), se busca la profundización en la estructura de relaciones que existe entre un grupo de elementos: personas, organizaciones, palabras, conceptos, entre otros. En la figura 1 se puede observar el impacto de las relaciones entre los actores involucrados en la red CUGE, para ello se tomó en consideración lo arrojado en el momento empírico de la investigación a través del cuestionario, donde los actores involucrados develaron la participación activa, al igual que el impacto de intensidad (fuerte, moderado, débil), de acuerdo a los vínculos asociados al intercambio y transacción de información y conocimiento, que tiene en el contexto su organización la influencia del comportamiento del resto de los actores e instancias organizacionales identificadas. Para el análisis de la red, siguiendo a Hanneman y Riddle (2005), se recurre a la herramienta metodológica que aporta el programa UCINET Versión 6.667, para analizar las propiedades de los actores dentro de la red. Al respecto se logra determinar: Densidad de la red, tamaño, grado, accesibilidad entre otros. Los principales hallazgos se muestran en la Figura 1.

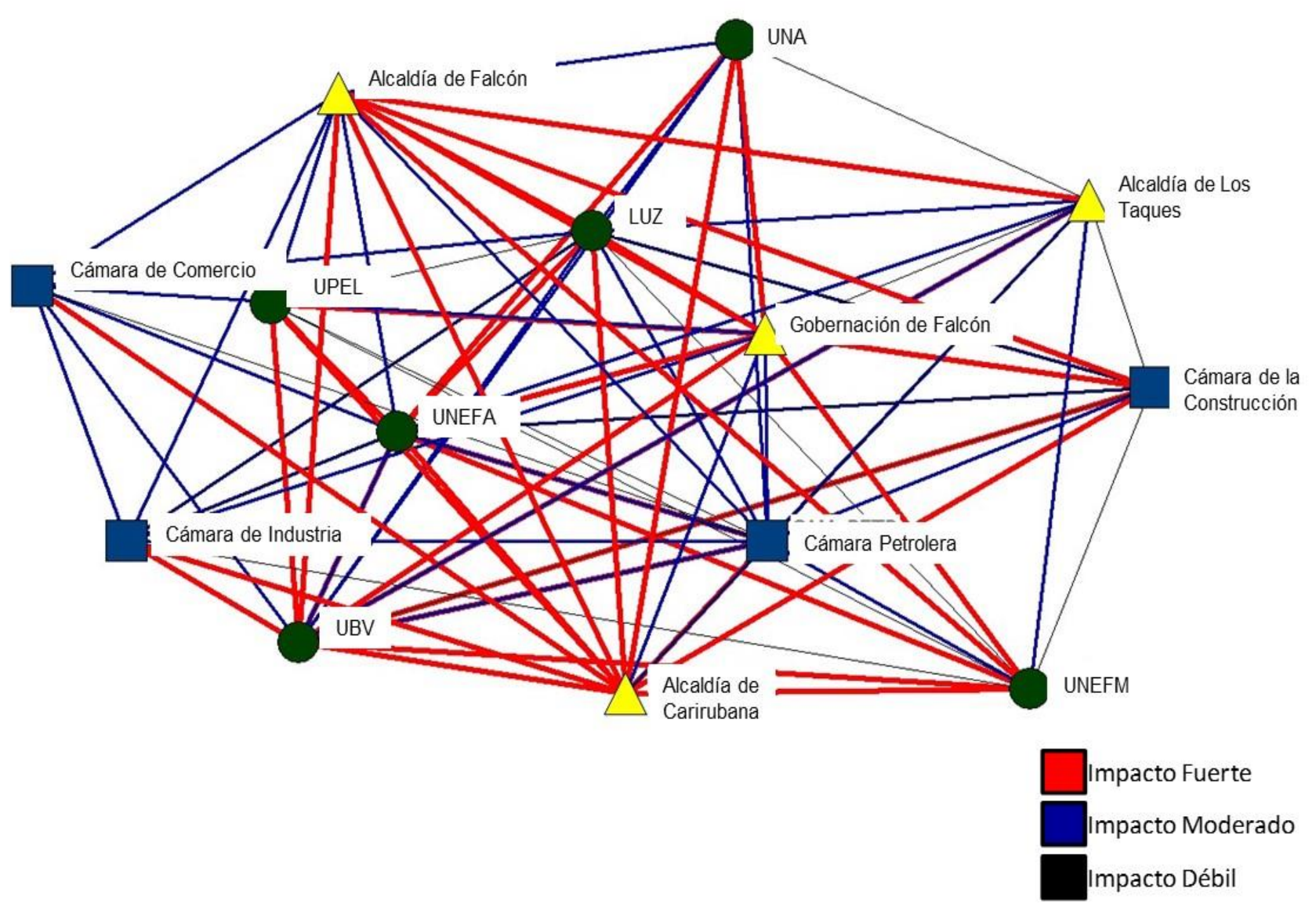

Fig. 1: Impacto de las relaciones entre los actores de la red CUGE

En la figura 1 se puede visualizar tanto el tamaño como las conexiones preferenciales entre los actores de la red CUGE, en este sentido se representa una red dirigida con un número limitado de actores conectados, se aprecian relaciones diádicas transitivas entre pares de actores con impacto fuerte, moderado o débil en sus aristas; los actores están personificados por círculos, triángulos y cuadrados respectivamente, los cuales hacen alusión al sector que representan, igualmente se puede apreciar una notable densidad de relaciones y conductas similares de relación entre los mismos. Ahora bien, tomando en consideración la disposición de las relaciones establecidas entre los nodos el comportamiento de la red es similar al de una red malla, donde, no existen vínculos competitivos, riesgo de interrupción entre vínculos mínimo y los actores mantienen relación permanente. A continuación y en busca de la precisión en relación a las propiedades de la red, se utilizan las medidas matemáticas de los indicadores del grafo. En la figura 2 se hace referencia al indicador densidad. 


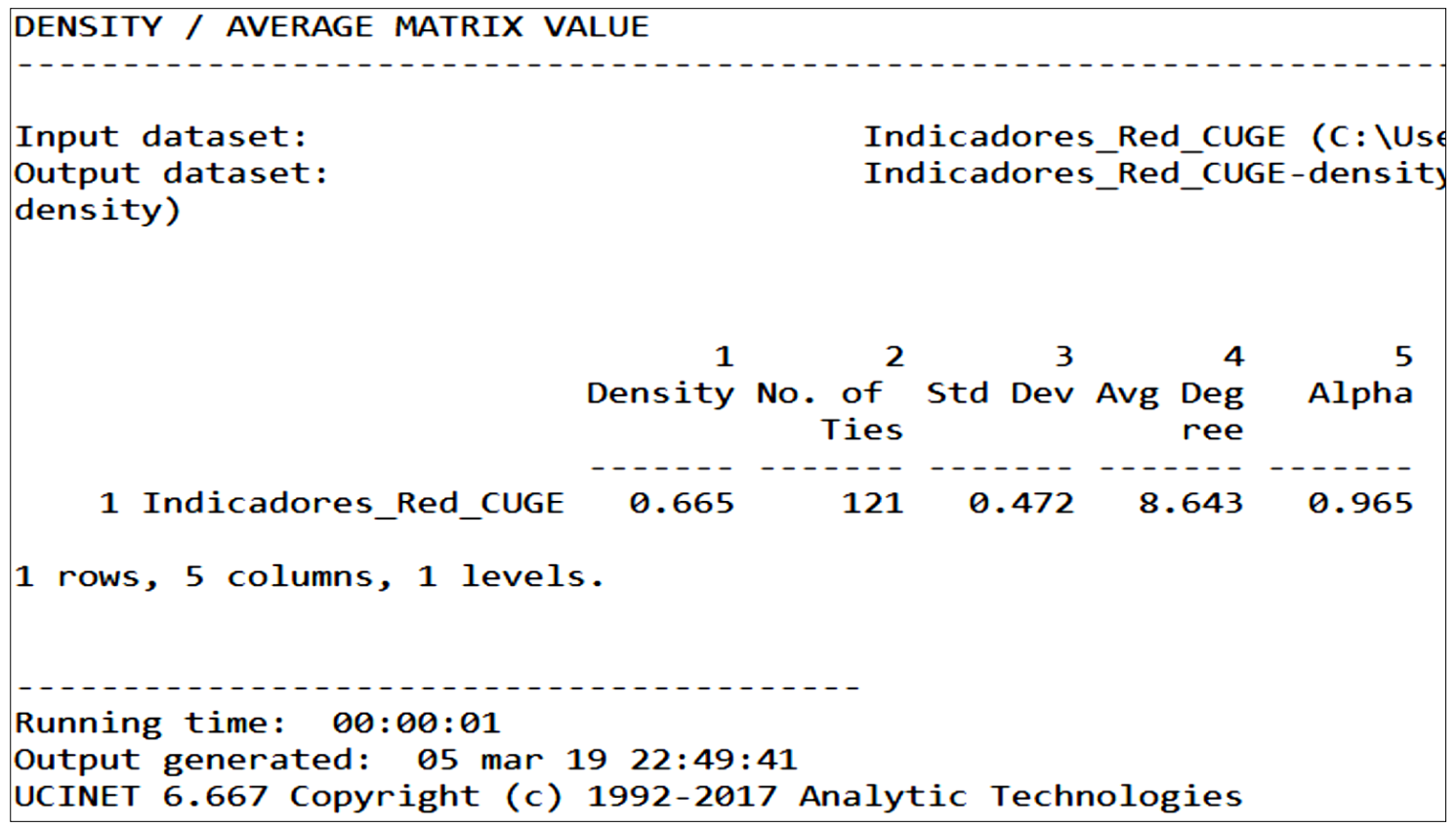

Fig. 2: Densidad de la red CUGE

En relación a la densidad de una red viene representada por la relación existente entre el número de lazos presentes y el número de lazos posibles; a tal efecto el resultado arrojado para la red CUGE considera una densidad de 0.665; que expresado en porcentaje seria $66.5 \%$, es decir los 121 vínculos observados en la red, representan el $66.5 \%$ de los vínculos posibles de toda la red (tamaño $\mathrm{N}=14$ ). Es importante también destacar el indicador del grado promedio de la red, para este caso fue de 8.643; es decir, cada uno de los 14 actores que componen la red tienen 8.6 vínculos en promedio. De Rolt et al. (2017) consideran que la consolidación de una red está relacionado a la dinámica de la relaciones de los actores. Se busca por tanto mantener una densidad relativamente elevada para garantizar fluidez en los intercambios de recursos, información y beneficiar la coordinación. En correspondencia a la densidad como propiedad estructural, surgen los grados de centralidad para cada uno de los actores, que se muestran en la Figura 3.

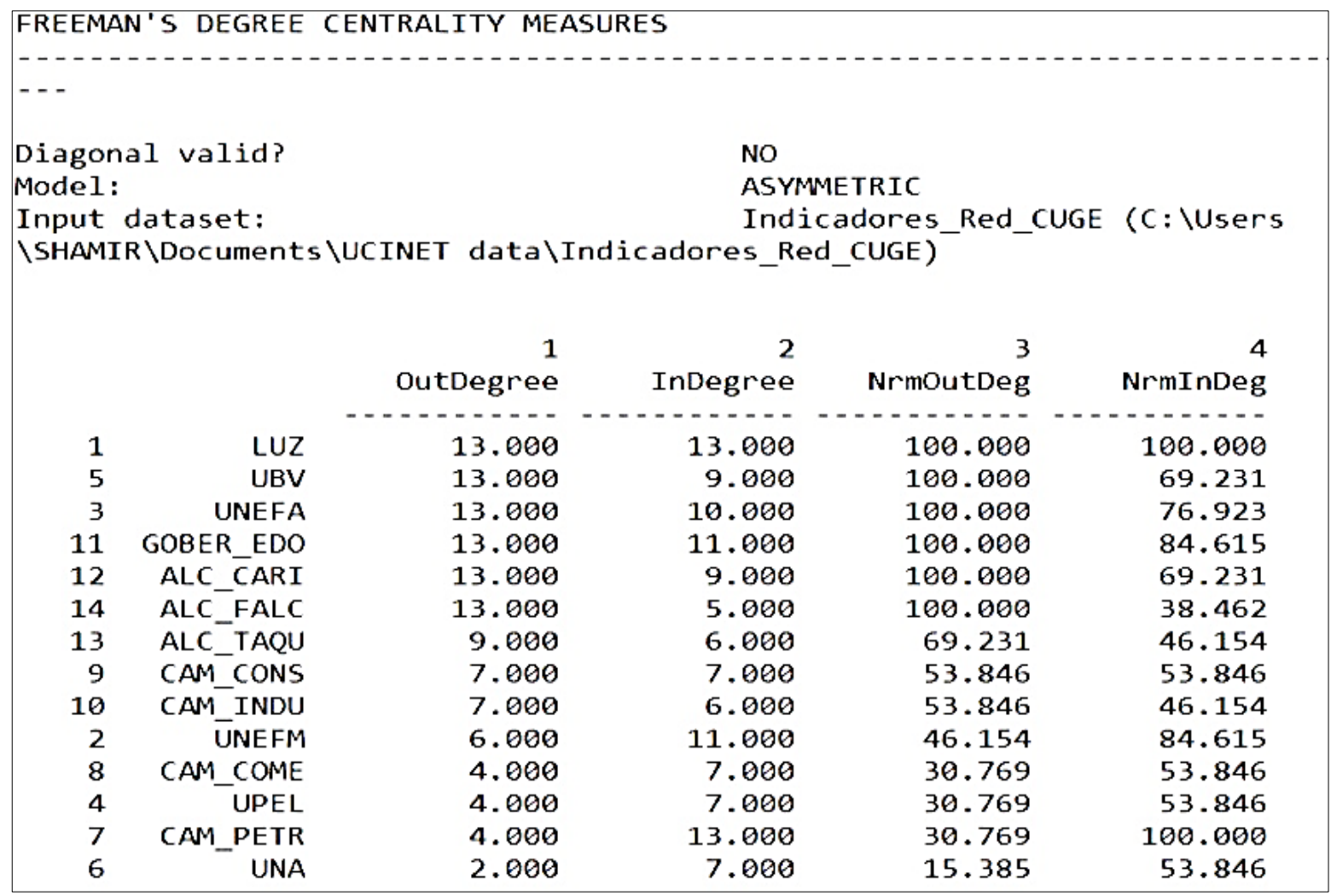

Fig. 3: Centralidad de grado por actor de la Red CUGE 
Como se puede apreciar, el conjunto de resultados está conformado por cuatro columnas, en el siguiente orden: grado de salida (OutDegree), grado de entrada (In Degree), grado de salida normalizado (NrmOutDegree) y, grado de entrada normalizado (NrmOutDegree). En este sentido y considerando que el orden de los nodos está presentado considerando el grado de salida, de mayor a menor, los nodos: LUZ, UBV, UNEFA, GOBER_EDO, ALC_CARI, ALC_FALC; son los de mayor grado de salida (refiriendo a los trece actores restantes como sus fuentes de información), importante mencionar que el resto de los nodos tienen grados de salida en menor proporción siendo UNA la que menos salida posee con 2 actores como fuente de información. Por otro lado, los actores más referidos y que, por tanto, son de gran importancia para la red, está presentado por LUZ y CAM_PETR con 13 grados de entrada respectivamente, seguido por UNEFM y GOBER_EDO con 11 grados de entrada cada uno. En lo referente a las dos columnas restantes, que representan los indicadores normalizados, son básicamente densidades individuales, es decir, la proporción (expresada en porcentaje) de vínculos que cada actor tiene con respecto a las posibles. Para el caso que nos ocupa, la red CUGE es de 14 nodos, entonces cada uno de los actores podría vincularse a cada uno de los otros 13 veces; Se puede señalar entonces, que para los grados de salida de UNEFM son 6 de 13 posibles, por lo tanto, su grado de salida normalizado es de 46,15\%, y así para el resto de los actores. Este mismo análisis aplica para el grado de entrada de los nodos. Gutiérrez y Narváez (2017) consideran que los actores que ocupan posiciones centrales pueden controlar la generación de contactos y el flujo de información, además incidir en la opinión y conducta de otros.

El diseño parte de la existencia de una cultura reticular, con diversidad de interacciones y flujos, donde los actores confluyan desde sus diferentes perfiles de participación y responsabilidades asumidas, donde existan mecanismos que permitan el acercamiento de nuevos actores, así como sólidos mecanismos de comunicación e información, lo que permita potenciar sus capacidades y fortalezas en la búsqueda de solución de problemas que existen en su entorno. El abordaje plantea a la red CUGE la identificación de actores, relaciones y estructura morfológica con especial atención en densidad, y actores centrales de la red.

\section{CONCLUSIONES}

Basado en el estudio, los resultados, su discusión y análisis, se pueden obtener las siguientes conclusiones principales.

i) Se valida la tesis de que las relaciones establecidas entre la universidad, el gobierno y la empresa propician la creación de redes formales de cooperación, para una gestión eficiente de las prácticas profesionales.

ii) Se confirma la influencia que tienen las relaciones previas entre los diferentes actores sociales para su conformación

iii) Las relaciones previas se ven influenciada por las condiciones académicas, tecnológicas e industriales de la zona de su radio de acción.

iv) Se encontró que existen actores con características reticulares deseables, los cuales tienen implicaciones importantes en el manejo de información, conocimiento y gestión de la red CUGE

\section{REFERENCIAS}

Aguilar-Gallegos, N., Martínez-González, E., y otros cuatro autores. Análisis de Redes Sociales para Catalizar la Innovación Agrícola: de los Vínculos Directos a la Integración y Radialidad, Estudios gerenciales, 32, 197-207 (2016)

Aguirre, J., Actores, Relaciones y Estructuras: Introducción al Análisis de Redes Sociales, Hologramática - Facultad de Ciencias Sociales - UNLZ - Año XI, II(20), 161-187 (2014)

Barrios-Hernández, K., y Olivero-Vega, E. Relación Universidad-Empresa-Estado. Un Análisis desde las Instituciones de Educación Superior de Barranquilla-Colombia, para el desarrollo de su capacidad de innovación, http://doi.org/10.4067/S0718-50062020000200021, Formación Universitaria, 13(2), 21-28, (2020)

Bonal, X. La Educación en Tiempos de Globalización: ¿Quién se Beneficia?, Educación Social Campinas, 30(108), 653671 (2009).

Borgatti, S., Everett, M., y Freeman, L. Ucinet 6.667 for Windows: Software for Social Network Analysis, Harvard, MA: Analytic Technologies (2002)

Bravo, O., Marín-González, F., y Carrera, M. Redes Interorganizacionales y Desarrollo Local, Opción, 29(70), 86-103 (2013)

De Rolt, C., Da Silva, D., y García, F. Network Analysis as a Management Tool for Inter-organizational Projects, Gestão \& Produção, 24(2), 266-278 (2017)

Espinoza, R., y Marín-González, F. Redes de investigación Transdisciplinar Tecnocientífico en Contextos Reticulares / Networks of Transdisciplinary Technoscientific Research in Reticular Contexts. Utopía y Praxis Latinoamericana, 24 (87) 173-193 (2019). 
Freeman, L.C., The Development of Social Network Analysis: A Study in the Sociology of Science, Empirical Press, BookSurge. (2004)

Gardner, P., y Tyson, V. Diversity, Work and Educatión: A Case for Experiential Learning During Times of Change, The Journal of Cooperative Education, 29, 11-25 (1994)

Gulati, R. Aliances and Networks, Strategic Management Journal, 19(4) 293-317 (1998)

Gutiérrez, M., y Narváez, M. Aproximación Teórica a las Redes de Cooperación en el Marco de la Actividad Turística, Innova Research Journal, 2(9), 86-97 (2017)

Hanneman, R.A., y Riddle, M. Introduction to Social Network Methods. Riverside, CA, University of California, (2005)

Hernández, de V. J., y Chumaceiro, A. Acercamiento Histórico a la Participación Ciudadana en Venezuela, Revista de Ciencias Sociales, 24 (2), 56-67 (2018)

Herrera-Núñez, Y. A., y González-Campos, J. A. Redes de Dependencia entre Estrategias de Aprendizaje y Perfiles de Estudiantes de Desempeño Académico Medio y Alto en el Contexto de la Educación Superior en Chile, https://doi.org/10.4067/S0718-50062019000400027, Formación Universitaria, 12(4) 27-38 (2019). .

Larner, W. Globalising Knowledge Networks: Universities, Diaspora Strategies, and Academic Intermediaries. Geoforum, 59, 197-205 (2015).

Madeo, D., y Mocenni, C. Self-regulation versus social influence for promoting cooperation on networks, https://doi.org/10.1038/s41598-020-61634-7, Scientific Reports, 10(1), 30-48 (2020)

Maya-Jariego, I., y Holgado, D. Living in the Metropolitan Area. Correlation of Interurban Mobility with the Structural Cohesion of Personal Networks and the Originative Sense of Community, https://doi.org/10.1016/j.psi.2015.09.001, Psychosocial Intervention, 24(3), 185-190 (2015)

Marín-González, F., Talavera, R., Inciarte-González, A., y Avendaño-Villa, I. Redes de Cooperación CientíficoTecnológicas en Contextos Intersectoriales, https://doi.org/10.4067/S0718-07642019000300013, Información Tecnológica, 30(3), 13-24 (2019).

Marín-González, F., Cabas, L., y Paredes-Chacín, A. Formación Integral en Profesionales de la Ingeniería. Análisis en el Plano de la Calidad Educativa, https://doi/10.4067/S0718-50062018000100013, Formación Universitaria, 11(1), $13-24$. (2018).

Padrón, J. Paradigmas de Investigación en Ciencias Sociales. Un Enfoque Curricular. Espacio para un programa de formación de investigadores. Papel de Trabajo, Postgrado, USR. Caracas, (1992).

Peña, T., Castellano, Y., Díaz, D., y Padrón, W. Las Prácticas Profesionales como Potenciadoras del Perfil de Egreso: Caso: Escuela de Bibliotecología y Archivología de La Universidad del Zulia, Paradigma, 37(1), $211-230$ (2016)

Pérez González, J., Marín-González, F., y Mercado Porras, C., Factores asociados al desarrollo de las pasantías industriales en instituciones universitarias, Opción, 35(89-2), 509-549 (2019)

Romero, J., y Zavala, K. Planificación Estratégica Financiera en las Cadenas de Farmacias Familiares del Estado ZuliaVenezuela, Revista de ciencias sociales, 24(4), 9-25 (2018).

Sánchez, G., Pérez, J., y Picco, L. Redes de Conocimiento Basadas en la Gestión del Conocimiento: Creación y organización para docencia e investigación universitaria, Interamericana de Bibliotecología, 37(3), 215-225 (2014)

Sebastián, J. Las Redes de Cooperación como modelo organizativo y funcional para la I+D, Redes, 7(15), 97-111 (2000)

Tubin, D., y Levin-Rozalis, M. Interorganizational Cooperation: The Structural Aspect of Nurturing Trust, International Journal of Public Sector Management, 21(7), 704-722 (2008)

UNESCO., Declaración Mundial sobre la Educación Superior en el Siglo XXI: Visión y Acción y Marco de la Acción prioritaria para el Cambio y el Desarrollo de la Educación Superior. Conferencia Mundial sobre la Educación Superior, (1998)

Wasserman, S., y Faust, K. Social Network Analysis: Methods and Applications, Vol. 8, Cambridge University Press, Cambridge, Inglaterra (1994) 